\title{
A Reduced-Complexity Interacting Multiple Model Algorithm for Location Tracking in Heterogeneous Observation
}

\author{
Xiaoyan Fu \\ The College of Information Engineering, Capital Normal University, Beijing Advanced Innovation Center for Imaging \\ Technology, 100191, P.R. China \\ Yuanyuan Shang \\ The Beijing Key Laboratory of Electronic System Reliability Technology, Capital Normal University \\ Beijing, 100191, P.R. China \\ E-mail: fuxiaosg@163.com,syy@bao.ac.cn \\ www.cnu.edu
}

\begin{abstract}
This paper is devoted to the problem of state estimate of discrete-time stochastic systems. A low-complexity and high accuracy algorithm is presented to reduce the computational load of the traditional interacting multiple model algorithm with heterogeneous observations for location tracking. By decoupling the $\mathrm{x}$ and $\mathrm{y}$ dimensions to simplify the implementation of location, updated information is iteratively passed based on an adaptive fusion decision. Simulations show that the algorithm is more computationally attractive than existing multiple model methods.
\end{abstract}

Keywords: data fusion, interacting multiple model algorithm, location tracking, wireless sensor networks.

\section{Introduction}

Date fusion deals with the problem of how to extract and utilize useful information contained in multiple sets of data in order to estimate unknown parameters or processes $^{1}$, which has been widely applied in military and civilian fields, e.g., target tracking and localization, air traffic control, guidance and navigation, fault diagnosis, surveillance and monitoring. Estimation fusion is one of the important applications involving wireless sensor networks (WSNs), but the WSNs have very limited sensing range and communication bandwidth which restrict the application of the centralized signal processing method. In other words, not only are accurate positioning algorithms essential to useful location-estimation systems, but also to reduce energy consumption is worth developing lowcomplexity schemes for $\mathrm{WSNs}^{2}$.

State estimate problem of discrete-time Markovian jump linear system (MJLS) is always the focus of interest in the community of maneuvering target tracking. Multiple-model (MM) algorithms (such as generalized pseudo-Bayesian (GPB), interacting multiple model (IMM), variable-structure MM (SVMM)) are generally considered as mainstream approach to address this problem. Among them, IMM algorithm proposed by Blom $^{3}$ is most prevalent.

Nevertheless, the high accurate location estimation for maneuvering target based on the traditional IMM algorithm requires models interacting and inverse operations. The location data fusion algorithm with an IMM technique has high computational complexity, and 
direct implementation of the IMM algorithm may be too complex for practical systems. Moreover, local stations in WSNs may not be able to afford IMM estimation due to limited computational power ${ }^{4}$. Consequently, it would be useful to reduce the computational complexity of the IMM algorithm.

Our idea to deal with reduced-complexity state estimation is to reconstruct the IMM algorithm. In contrast to the existing reduced-complexity approach including: alpha-beta filtering, Kalman filtering, our proposed methods is based on a factor graph (FG) by decoupling the $x$ and $y$ dimensions to simplify the implementation of location and adaptive data decision for state estimation of a MJLS.

\section{Background}

\subsection{Model}

Consider the dynamic system of tracked target described with the state space form. The mathematical models on the target can be taken by the following Markov jump linear system:

$$
\begin{gathered}
x(k+1)=F_{j} x(k)+\Gamma_{j} \omega_{j}(k) \\
z(k)=H_{j} x(k)+v_{j}(k)
\end{gathered}
$$

where the state $x(k)$ is an n-dimensional vector, the observation $z(k)$ is an m-dimensional vector, and the subscript $j \in S=\{1,2, \ldots, S\}$ denotes the model. The matrix functions $F_{j}(\cdot) \Gamma_{j}(\cdot)$ and $H_{j}(\cdot)$ are known. The model-dependent process noise and measurement noise are assumed to be a Gaussian random process.

Let $M_{j}^{k}$ denotes the flight model $j$ at time $k$. The model dynamics are modeled as a finite Markov chain with known model-transitions probabilities from model $i$ at time $k-1$ to model $j$ at time $k$.

$$
\pi_{i j}=\operatorname{Pr} o b\left[M_{j}^{k} \mid M_{i}^{k-1}\right\}=P\left\{M_{j}^{k} \mid M_{i}^{k-1}\right\}
$$

\subsection{Operations of Factor Graphs}

In terms of the operations of FG operations, one advantage of multiplications is the distributive law. For example, $a \times b+a \times c=a \times(b+c)$, where the left-hand side involves three arithmetic operations, and the righthand side involves only two operations. Furthermore, according to the inherent distributed feature of the belief propagation algorithm to make the decomposition in accordance with prediction-correction recursion, the messages of the reliable information are processed and passed among the prediction phases and correction phases with the error propagation law ${ }^{5}$.

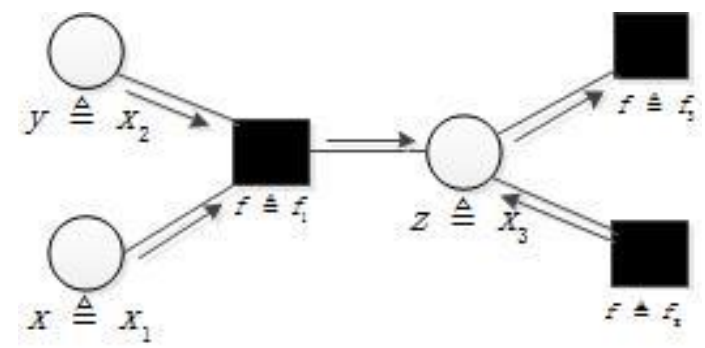

Fig. 1. Message passing from node to node

1) Message from Variable Nodes to Factor Nodes:

Assume that each message in the FG flows is a Gaussian PDF. A message from a variable node to a factor node is the product of incoming messages. For example, a variable node with two incoming messages is illustrated in Fig. 1, where a variable node is represented by a circle; a factor node is represented by a solid square. In fact, the message from a variable node to a factor node can be taken as the correction step in Bayesian filtering, where the mean is the estimated result based on data reliability for location estimation and tracking.

2) Message from Factor Nodes to Variable Nodes: For two continuous variables $\mathbf{x}$ and $\mathbf{y}$, the marginal density function of $\boldsymbol{y}$ is obtained by integrating the joint distribution over variable $\boldsymbol{x}$. This operation can be taken as the prediction step using the Bayesian filtering approach for location estimation and tracking.

\section{Proposed Location Tracking Algorithm}

This article focuses on location tracking approaches in terms of the $X$ and $Y$ groups individually, and the $X$ and $Y$ results of the state and the covariance equations are decoupled as individual input. Moreover, others heterogeneous observations can be viewed as individual input, and corresponding state estimation can be obtained individually. All estimations combined with data fusion algorithm, which can improve the accuracy of location estimation and tracking.

Given the computationally attractive feature of $\mathrm{FGs}^{6}$, this article proposes a location tracking scheme based on the FG approach and the error for location and speed estimations to implement the recursive Bayesian 
estimation and to reduce the computational load of the traditional IMM algorithm. Furthermore, decoupling the $X$ and $Y$ dimensions for different tracking groups also can reduce the computational complexity. Therefore, the 2-D problem is reduced to two 1-D problems. The 2-D problem can be represented by two independent main groups, the $X$-coordinate and the $Y$-coordinate groups.

According to the concept of undirected graph model, the representation related to estimate speed and location of the X-coordinate group is illustrated as the black color diagram in Fig. 2, where a correction node is illustrated by a circle; a prediction node is illustrated by a solid square.

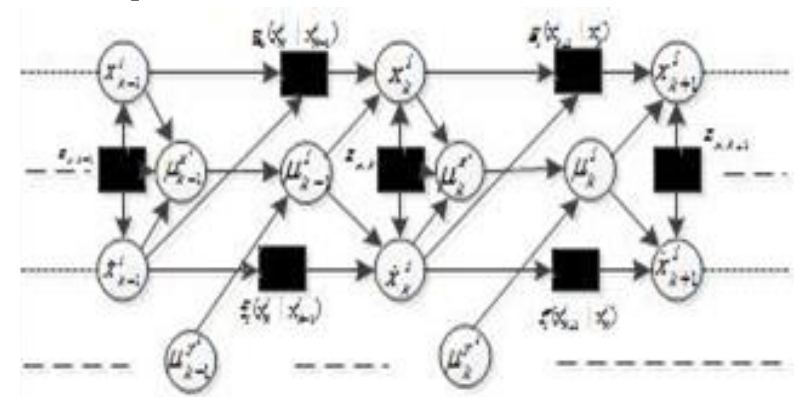

Fig. 2. Graph model of the data-fusion location estimation for $\mathrm{X}$-coordinate group from the $k$ th to $(k+1)$ th.

The measurement model based on IMM algorithm is the situation that the error propagation law can be applied to the linear dynamical system, and then the proposed IMM algorithm based on factor graphs (IMM-FG) for location estimation and tracking are illustrated in the following part.

Step 1: Calculate the mixed initial probability for the filter m-atched to model $M_{j}^{k}(j \in \mathbb{S})$

$$
\begin{aligned}
\mu_{i \mid j}(k \mid k) & \triangleq \mathrm{P}\left\{M_{i}^{k-1} \mid M_{j}^{k}, z_{0: k-1}\right\} \\
& =\frac{\mathrm{P}\left\{M_{j}^{k} \mid M_{i}^{k-1}, z_{0: k-1}\right\} \cdot \mathrm{P}\left\{M_{i}^{k-1} \mid z_{0: k-1}\right\}}{\mathrm{P}\left\{M_{j}^{k} \mid z_{0: k-1}\right\}} \\
& =\frac{\pi_{i j} \mu_{i}^{k-1}}{\bar{c}_{j}} \quad i, j \in \mathbb{S}
\end{aligned}
$$

where $\bar{c}_{j}$ is the normalization constant.

Step 2: Calculate the mixed initial state and corresponding covariance for the filter matched to model $M_{j}^{k}(j \in \mathbb{S})$

$$
\begin{aligned}
& x_{0 j}(\mathrm{k} \mid \mathrm{k}) \sim \sum_{i=1}^{s} \mu_{i \mid j}(k \mid k) N\left(\mathrm{x}_{i}^{k-1} ; \widehat{\mathrm{S}}_{x_{k-1}}, \widehat{\mathrm{V}}_{x_{k-1}}\right) \\
& \dot{x}_{0 j}(\mathrm{k} \mid \mathrm{k}) \sim \sum_{i=1}^{s} \mu_{i \mid j}(k \mid k) N\left(\dot{\mathrm{x}}_{i}^{k-1} ; \widehat{\mathrm{S}}_{\dot{x}_{k-1}}, \widehat{\mathrm{V}}_{\dot{x}_{k-1}}\right)
\end{aligned}
$$

Step3: Filtering $(j \in \mathbb{S})$

Prediction state

$$
\begin{aligned}
& x_{j}(k \mid k-1) \sim N_{\text {oper } 3}\left(x_{j}(k \mid k-1) ; \tilde{S}_{x_{0 j}(k \mid k)}, \tilde{V}_{x_{0 j}(k \mid k)}\right) \\
& \dot{x}_{j}(k \mid k-1) \sim N_{\text {oper } 2}\left(\dot{x}_{j}(k \mid k-1) ; \tilde{S}_{\dot{x}_{0 j}(k \mid k)}, \tilde{V}_{\dot{x}_{0 j}(k \mid k)}\right)
\end{aligned}
$$

Correction state

$$
\begin{aligned}
& x_{j}^{k} \sim N_{\text {oper } 1}\left(x_{j}^{k} ; \widehat{\mathrm{S}}_{x_{j}(\mathrm{k} \mid \mathrm{k}-1)}, \widehat{\mathrm{V}}_{x_{j}(k \mid k-1)}\right) \\
& \dot{x}_{j}^{k} \sim N_{\text {oper } 1}\left(\dot{x}_{j}^{k} ; \widehat{\mathrm{S}}_{\dot{x}_{j}(\mathrm{k} \mid \mathrm{k}-1)}, \widehat{\mathrm{V}}_{\dot{x}_{j}(k \mid k-1)}\right)
\end{aligned}
$$

Step4: Combine the state estimates and corresponding covariance according to the updated weights

$$
\begin{aligned}
& x_{I}(\mathrm{k}) \sim \sum_{j=1}^{s} \mu_{j}^{k} N\left(\mathrm{x}_{j}^{k} ; \widehat{\mathrm{S}}_{x_{k}}, \widehat{\mathrm{V}}_{x_{k}}\right) \\
& \dot{x}_{I}(\mathrm{k}) \sim \sum_{j=1}^{s} \mu_{j}^{k} N\left(\dot{\mathrm{x}}_{j}^{k} ; \widehat{\mathrm{S}}_{\dot{x}_{k}}, \widehat{\mathrm{V}}_{\dot{x}_{k}}\right)
\end{aligned}
$$

Updated weight of model $M_{j}^{k}$ is

$\mu_{j}^{k}$

$$
\begin{aligned}
& \triangleq \mathrm{P}\left\{M_{j}^{k} \mid Z^{k}\right\} \\
& =\text { decision }\left[\mathrm{P}\left\{M_{j}^{k} \mid z_{1, k}, s_{1, k}\right\}, \mathrm{P}\left\{M_{j}^{k} \mid z_{2, k}, s_{2, k}\right\},\right. \\
& =\text { decision }\left[\mu_{x, j}^{k}, \mu_{y, j}^{k}, \mu_{*, j}^{k}\right] \\
& \text { where } \\
& \qquad \mu_{x, j}^{k}=\frac{\mathrm{P}\left\{z_{x, k} \mid M_{j}^{k}, z_{0: k-1}\right\} \cdot \mathrm{P}\left\{M_{j}^{k} \mid z_{0: k-1}\right\}}{c} \\
& \qquad=\frac{N\left[z_{x, k} ; \widehat{\mathrm{S}}_{x_{0 j}(\mathrm{k} \mid \mathrm{k})}, \widehat{\mathrm{V}}_{x_{0 j}(\mathrm{k} \mid \mathrm{k})}\right] \cdot \bar{c}_{j}}{c}
\end{aligned}
$$$$
=\operatorname{decision}\left[\mathrm{P}\left\{M_{j}^{k} \mid z_{1, k}, s_{1, k}\right\}, \mathrm{P}\left\{M_{j}^{k} \mid z_{2, k}, s_{2, k}\right\}, \mathrm{P}\left\{M_{j}^{k} \mid s_{*, k}\right\}\right]
$$

It's worth mentioning that decision [.] refers to a decision-making or data fusion process, for example, mean[.], $\max [],. \min [$.$] or more intelligent decision,$ different location and tracking problems can adopt different data fusion for better tracking accuracy.

\section{Simulation Results}

In this section, we evaluate the performance of IMMFG algorithm, and the classical IMM algorithm using the target tracking example. The results are obtained from 100 Monte Carlo runs. The $X$ position and velocity RMSE are shown in Fig.3 and Fig.4, respectively. The $Y$ position and velocity RMSE are omitted due to limited space. It can be found that accuracy estimate of the 
IMM algorithm as nearly as the proposed IMM-FG algorithm in almost time. Although IMM-FG algorithm has a higher position estimate error than IMM algorithm during the second maneuvering (time samples 61-66), the estimate error converges to zero within a short time. The occurrence of this phenomenon maybe due to that the IMM-FG algorithm pays more attention to the rapid change of the velocity during the high maneuvering. On the whole, the estimation accuracy of the IMM-FG algorithm is almost the same with the classical IMM algorithm, but the computing time is short. It can be verified through the comparison computing time between IMM and IMM-FG given in Table 1.

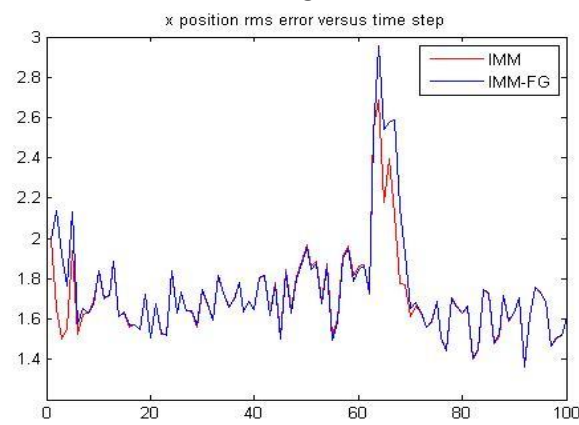

Fig. 3. X position RMSE versus time step.

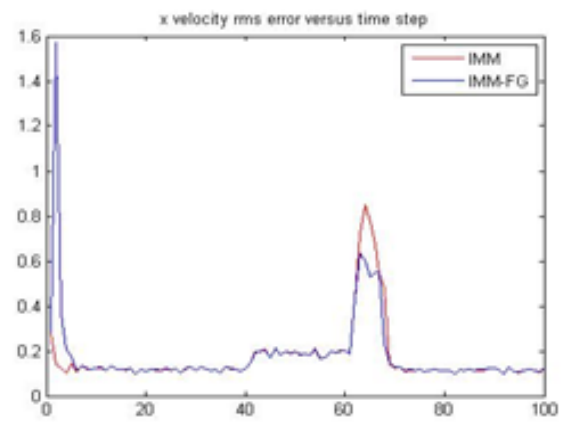

Fig. 4. X speed RMSE versus time step

Table1. Computing time between IMM and IMM-FG

\begin{tabular}{|l|l|l|}
\hline & IMM & IMM-FG \\
\hline 50 Monte Carlo runs & 1.3767 & 1.1228 \\
\hline 100 Monte Carlo runs & 2.4825 & 2.1691 \\
\hline
\end{tabular}

\section{Conclusion}

The conclusion comes here. In the paper, IMM-FG algorithm is presented for maneuvering target tracking.
It is principally similar to the popular IMM algorithm. The difference lies in the use of filtering. To avoid the inverse of covariance matrix and reducing the complexity of tracking algorithm, the FG algorithm and the idea of decoupling the $X$ and $Y$ dimensions to simplify the implementation of location estimation and tracking is induced to construction of IMM algorithm. Computer simulations indicate that the IMM-FG algorithm has almost the same tracking accuracy with the IMM algorithm.

\section{Acknowledgements}

This work was supported by the NSFC (11178017, 61373090, 61303104, 61203238) and the BNSFC (4132014).

\section{References}

1. D.Jeon, E. Yeonju and K. Hyounkyoung, "Estimation fusion with radar and ADS-B for air traffic surveillance", Information Fusion, 16th International Conference on, 2013, pp. 1328-1335

2. C.L.Wang, Y.S.Chiou and F.Tsai,"Reduced-complexity tracking scheme based on adaptive weighting for location estimation ", IET communications, 2012, pp. 673-684.

3. Blom, H.A.P., Bar-Shalom, Y.,"The interacting multiple model algorithm for systems with Markovian switching coefficients", IEEE Transactions on Automatic Control, 1988, 33, (8), pp. 780-783.

4. Y.X. Gao, X.R. Li and Z.S. Duan, "Estimation Fusion for Markovian Jump Linear System via Data Transformation", IEEE Transactions on Aerospace and Electronic Systems, vol. 50, no. 1, pp. 240-253, Jan.2014

5. Y.S. Chiou and F. Tsai, "A Reduced-Complexity DataFusion Algorithm Using Belief Propagation for Location Tracking in Heterogeneous Observations", IEEE Transactions on Cybernetics, vol. 44, no.6, pp. 922-935, Jun. 2014

6. C.L. Wang, Y.S. Chiou, and F. Tsai, "Reducedcomplexity tracking scheme based on adaptive weighting for location estimation", IET Communication,vol. 7, no. 7, pp. 673C684, May. 2013

7. Y.-S. Chiou, F. Tsai, C.-L. Wang, and C.-T. Huang, 'A reduced-complexity scheme using message passing for location tracking,'EURASIP J. Adv. Signal Process ., vol 2012, Jun. 2012.

8. Ho, T.J., Farooq, M.: 'Comparing an interacting multiple model algorithm and a multiple-process soft switching algorithm'.Information Fusion, 2000. pp. 17-24. 